Conclusions Deriving causal treatment effects using observational health data has proven to be problematic. We demonstrate that in contrast to IV based estimates, conventional OLS methods fail to estimate casual effects as indicated by RCTs.

\section{Childhood and mental health 009 CHRONIC ILLNESS AND EMOTIONAL AND BEHAVIOURAL
STRENGTHS AND DIFFICULTIES IN IRISH CHILDREN}

doi:10.1136/jech.2010.120956.9

${ }^{1,2} \mathrm{U}$ Reulbach, ${ }^{1} \mathrm{~T}$ O'Dowd, ${ }^{3} \mathrm{C}$ McCrory, ${ }^{3} \mathrm{R}$ Layte. ${ }^{1}$ Department of Public Health and Primary Care, Trinity College Centre for Health Sciences, Dublin, Republic of Ireland; ${ }^{2}$ HRB Centre for Primary Care Research, Dublin, Republic of Ireland; ${ }^{3}$ Economic and Social Research Institute (ESRI), Dublin, Republic of Ireland

Objective Childhood chronic illness can have a high impact on the child's quality of life. The foundations of health are established in early life, and are shaped by biological, psychosocial, spiritual and environmental processes and influences. The objectives of this presentation are twofold: firstly to describe the prevalence of chronic illness in 9 -year-olds in Ireland, secondly to illustrate how chronic illness influences the psychological and social development of the children. Methods Analysis was based on data of 8570 9-year old children, and their families who participated in Growing Up in Ireland-the National Longitudinal Study of Children. The sample was generated through the primary school system. A representative sample of 910 schools participated; the sample of children and their families was randomly selected from within the schools. Questionnaires were administered in schools; and after completion of this phase, the project interviewers visited the families of the 9-year olds in their homes and administered core questionnaires to the Study Child and his/her carers who provided either home-based or centre-based care on a regular basis.

Results The overall prevalence of chronic illness reported by mothers among the 9-year old cohort was 11\% (gender-specific prevalence for boys: $13 \%$, and significantly lower for girls: $10 \%)$. Respiratory illnesses accounted for almost half (46\%) of all chronic illnesses, followed by mental and behavioural conditions with 19\% (high gender dysbalance: reported for boys in $24 \%$, for girls in $12 \%$ ). Children with a reported chronic illness had significantly more emotional, conduct, hyperactivity, peer-based and prosocial difficulties when compared with children without a reported chronic illness. Abnormal scores, based on the Strengths and Difficulties Questionnaire were found in $20 \%$ in children with a chronic disease, and in $5.5 \%$ in children without a chronic disease. Socio-economic Status was associated with poorer health outcomes. Furthermore, primary care givers' views and perceptions regarding the chronic illness of the child were found to be a significant factor on child strengths and difficulties in a multivariate model.

Conclusions The majority of 9-year old children can be assessed as healthy. The most common chronic illness in this large cohort was respiratory disease and overall, chronic conditions were found to have a negative impact on the child's emotional and social state.

\section{PARENTAL SEPARATION AND PSYCHOLOGICAL DISTRESS IN EARLY ADULTHOOD: HAS THE EFFECT REDUCED OVER TIME? EVIDENCE FROM TWO BRITISH BIRTH COHORT STUDIES}

doi:10.1136/jech.2010.120956.10

R Lacey, M Bartley, H Pikhart, N Cable, M Stafford. Department of Epidemiology and Public Health, University College London, London, UK

Background An association between parental separation occurring during childhood and psychological distress has been shown many times. UK divorce rates have increased rapidly since the mid-twentieth century. The "reduced effect hypothesis" suggests that the effect of parental separation may have reduced over time as separation has become more common and consequently less stigmatising. Previous studies have looked at this using outcomes of educational attainment, psychological distress in mid-adulthood and receipt of welfare benefits, finding that the effect has not reduced over time; however the effect upon psychological distress in early adulthood has not yet been investigated.

Objective To examine whether the effect of parental separation occurring during childhood on psychological distress in early adulthood has reduced over time, and whether this differs for men and women.

Data, participants and variables This study uses data on 9064 and 6906 participants of the 1958 National Child Development Study (NCDS) and 1970 British Cohort Study (BCS70), respectively. Five sweeps of each study were used - birth, age 5/7, age 10/11, age 16 and age $23 / 26$ years. Parental separation was measured from 0 to 16 years and psychological distress was measured using Rutter's Malaise Inventory at age 23 (NCDS) or age 26 (BCS70), treated as a binary variable $(0-7=$ no distress, $8-24=$ distress $)$. Mental illness in the family was treated as a confounder.

Statistical methods Logistic regression was used to test the association between parental separation and psychological distress in either cohort, both unadjusted and adjusted for confounders, using a pooled data set of both cohorts. Period changes were assessed by testing a cohort-separation interaction. Analyses were conducted separately for men and women.

Results $9.4 \%$ of NCDS participants experienced parental separation in comparison to $21.0 \%$ of BCS70 participants. After adjusting for confounders parental separation increased the odds of reporting psychological distress in NCDS men (OR 2.18, 95\% CI 1.34 to 3.54 ) and women (OR 1.48, 95\% CI 1.05 to 2.08) and this did not differ by gender $(p=0.11)$. Parental separation was not associated with psychological distress in BCS70 adjusted analyses for men or women. However cohort-separation interactions were not statistically significant (men: $p=0.11$, women: $p=0.43$ ).

Conclusions Despite finding an effect of separation in the NCDS and not in the BCS70, the cohort-separation interactions tested were statistically insignificant. The findings of this study therefore imply that the impact of parental separation has not changed over time ("reduced effect hypothesis" is not supported) and that men and women are affected equally.

\section{GENDER DIFFERENCES IN THE EFFECT OF BREAST FEEDING ON ADULT PSYCHOLOGICAL WELL-BEING}

doi:10.1136/jech.2010.120956.11

N Cable, M Bartley, A McMunn, Y Kelly. Department of Epidemiology and Public Health, University College London, UK

Objective To examine the changes in the social distribution of breast feeding and its effect on the psychological well-being of adults via the pathway of childhood psychological health.

Design Prospective cohort study.

Setting We used two British Birth Cohort Studies: National Child Developmental Study (NCDS, born in 1958) and 1970 British Birth Cohort Study (BCS70, born in 1970).

Participants Those who completed information on childhood data (breast feeding, mother's educational level, parenthood at birth, presence of older sibling, and psychosocial adjustment) and midadulthood (psychological ill health and self-efficacy) were included in this study (NCDS: $\mathrm{N}=7750$; BCS70: $\mathrm{N}=6492$ ).

Main outcome measure Childhood psychosocial adjustment was measured by the Bristol Social Adjustment Guides for the NCDS (collected at age 11) and the Rutter scale graded by a teacher for the 
BCS70 (collected at age 10). Adult psychological well-being (NCDS=age 33; BCS70=age 30) was indicated by psychological ill health and self-efficacy. Adult psychological ill health was indicated by the Malaise Inventory with a cutoff point of 7 or above. Selfefficacy was derived from the response to questions asking the study participants about their perceived level of control over their life.

Methods A dichotomised index of childhood adversity was created after tabulating information about parenthood, mother's age, mother's education, and presence of older siblings. The effect of breast feeding on childhood psychosocial adjustment and adult psychological well-being was examined using logistic regression. Men and women were analysed separately and the effects of breast feeding on the outcomes were adjusted for confounders.

Results Findings showed that the magnitude of the effect of breast feeding on adult psychological well-being is larger in women than in men. After accounting for the effect of childhood social adversity, breast feeding promoted psychosocial adjustment during childhood in girls in NCDS (OR 1.25, 95\% CI 1.05 to 1.48) and in BCS70 (OR $1.44,95 \%$ CI 1.15 to 1.81 ), but not in boys.

In adulthood, being breast fed at birth was associated with higher self-efficacy (OR 1.31, 95\% CI 1.06 to to 1.61) and lower risk of psychological ill health (OR $0.76,95 \%$ CI 0.61 to to 0.96 ) in women in BCS70 only. However, no significant interaction effect was found between breast feeding and childhood social adversity.

Conclusion Although breast feeding did not moderate the negative effect of childhood social adversity on childhood or adulthood outcomes in this study, findings suggest that the practice of breast feeding can be important for women's psychological well-being throughout the lifecourse.

\section{BREAST FEEDING AND BEHAVIOURAL DEVELOPMENT IN CHILDREN: FINDINGS FROM THE MILLENNIUM COHORT STUDY}

\section{doi:10.1136/jech.2010.120956.12}

${ }^{1} \mathrm{~K}$ Heikkilä, ${ }^{2} \mathrm{~A}$ Sacker, ${ }^{3} \mathrm{Y}$ Kelly, ${ }^{4} \mathrm{M} \mathrm{J}$ Renfrew, ${ }^{1} \mathrm{M}$ A Quigley. ${ }^{1}$ National Perinatal Epidemiology Unit, University of Oxford, UK; ${ }^{2}$ Institute for Social and Economic Research, University of Essex, UK; ${ }^{3}$ Department of Epidemiology and Public Health, University College London, UK; ${ }^{4}$ Mother and Infant Research Unit, University of York, UK

Aim Our aim was to examine whether breast feeding is associated with behavioural development in children aged 5 years.

Methods We used data from a large, prospective, nationally representative UK cohort, the Millennium Cohort Study. Breast feeding was ascertained from parent-interviews at baseline and child behaviour from parent-rated outcome, the Strengths and Difficulties Questionnaire (SDQ). Our analyses included 10037 mother-child pairs with data on breast feeding, SDQ and potential confounders. 9525 of the children were born at term and 512 were preterm. We used logistic regression models to investigate the associations of breast feeding duration with abnormal parent-rated SDQ total and sub-scores at age five in term and preterm children separately.

Results Overall, abnormal SDQ scores were less common in breast fed than formula-fed children. Term children breast fed for four months or longer had lower odds of an abnormal total SDQ score (multivariable-adjusted $\mathrm{OR}$ compared to never breast fed children: $0.65,95 \%$ CI 0.52 to 0.82 ). This effect was similar for all the SDO sub-scores. In preterm children prolonged breast feeding was generally associated with lower odds of abnormal SDQ total and sub-scores but the effect estimates were imprecise. The associations between exclusive breast feeding and abnormal SDQ scores were similar to those of any breast feeding and abnormal SDQ scores.

Conclusions Our findings suggest that, at least in term children, prolonged breast feeding is associated with having fewer parentrated behavioural problems at the age of 5 years.

\section{(Un)employment and health}

\section{HAS YOUR WORK WORKED YOU TOO HARD: AN EXAMINATION OF WORK HISTORY, PRESENT FUNCTIONAL LIMITATIONS AND REDUCED ACTIVITIES OF LIVING IN A COHORT OF THE IRISH GENERAL POPULATION}

doi:10.1136/jech.2010.120956.13

V J C Mc Carthy, I J Perry, B A Greiner. Department of Epidemiology and Public Health, University College Cork, Cork, Republic of Ireland

Objective In the present paper, we examine the association between manual work, resultant functional limitations and reduced ability to carry out daily activities of living (ADL), in older age. We hypothesise that manual workers as opposed to non-manual workers suffer, in older age, functional limitations and reduced ability to carry out ADL. This study is of great importance owing to our ageing Irish population and an ailing health service.

Methods A 10 year follow up study was conducted on a cohort of the general population (59-80 year olds) in the Republic of Ireland. Specific data on physical measurements, marital status, educational attainment, work history, functional status and $\mathrm{ADL}$ was collected on 357 study participants. Work history data focused on the job the participant had done for the longest period of time, paid or unpaid. Each participant was then asked if they described this work as manual or non-manual. Functional limitations and ADL were assessed using validated scales.

Results Just over half the sample were female (53\%) with $44 \%$ $(n=150)$ retired (median $=9$ (5.14) years). Over $60 \%$ of the participants were, or had been engaged in manual work with this percentage higher in males, albeit non-significant (68\% vs $58 \%$, $\mathrm{p}=0.08$ ). $20 \%$ of the total sample had complete function with a higher proportion of non-manual as opposed to manual workers $(p=0.07)$ with no limitations. Almost three quarters of the sample had functional limitations and less than one fifth were classified as having an $\mathrm{ADL}$ disability.

Using linear regression, manual workers were significantly more likely to have functional limitations even after adjustment $(B=0.85$, $\mathrm{SE}=0.30, \mathrm{p}=0.01)$ for socio-demographic factors. When stratified by age, manual work remained significantly associated with functional limitations in the 60-69 year olds $(B=0.66, S E=0.34, p=0.05)$ and in the $70-80$ year olds $(B=1.13, S E=0.50, p=0.03)$. For the older age group, males had a decreased risk of functional limitations independent of work type $(B=-1.06, S E=0.47, p=0.03)$.

There was no significant association between manual work and reduced activities of daily living either in the unadjusted or adjusted model.

Discussion Functional limitations, in an older population, are related to the type of work they carried out. Good health surveillance for manual workers at a young age can identify limitations early. Initiatives such as work organisation, education and promotion of best work practices with regard to manual work can reduce functional limitations in older age.

\section{HOW DO MUSCULOSKELETAL PAIN, AVOIDANT COPING AND SICKNESS ABSENCE RELATE TO EACH OTHER?}

\section{doi:10.1136/jech.2010.120956.14}

U Christensen, C Ørsted Hougaard, K Thielen, E Nygaard, R Lund. Department of Public Health, Section of Social Medicine, University of Copenhagen

Background Musculoskeletal pain is common and causes extensive sickness absence and work disability in many industrialised countries. The prevalence of musculoskeletal morbidity increases by decreasing socio-economic position and it appears that people with a disadvantaged social situation are more vulnerable to the 